\title{
X-Ray, Optical, and Near-IR Long-Term Monitoring of AGN
}

\author{
Paulina Lira ${ }^{1}$, Patricia Arévalo ${ }^{2,3}$, Phil Uttley ${ }^{4}$, Ian $\mathrm{McHardy}^{4}$, \\ and Elme Breedt ${ }^{4}$ \\ ${ }^{1}$ Departamento de Astronomía, Universidad de Chile, Casilla 36D Santiago, Chile \\ ${ }^{2}$ Max-Planck-Institut für Astrophysik, Karl-Schwarzschild-Str. 1, D-85748 Garching, Germany \\ ${ }^{3}$ Departamento Ciencias Físicas, Universidad Andrés Bello, Av. República 252, Santiago, Chile \\ ${ }^{4}$ School of Physics and Astronomy, University of Southampton, Southampton SO17 1BJ, UK
}

\begin{abstract}
We are conducting long-term monitoring of several AGN with the aim of understanding the interplay between the emitting regions around the central black hole as well as the physics of the accreting flow. Here we present results for two of our sources, NGC 3783 and MR 2251-178, with strong evidence for disk-driven variability and the existence of cold accretion disks, where near-IR emission can be clearly detected from a region close to the black hole.
\end{abstract}

Keywords. accretion disks, galaxies: active, galaxies: individual (NGC 3783), galaxies: individual (MR 2251-178), X-rays: galaxies, infrared: galaxies

\section{Introduction}

Variability was established very early as a trademark of AGN. Variability has also been used as a key tool to derive physical properties in these systems: characteristic time scales allow us to infer sizes of the emitting regions, lags between the ionizing continuum and the response from line-emitting clouds have been used to determine black hole masses, and multiwavelength light curves have been used to study the physical and geometrical connections between the different regions around the central engine.

The scenario accepted early on for the interplay of the emiting regions is that variability is driven by the emission from the X-ray corona located close to the central black hole (e.g., Krolik et al. 1991; Collier et al. 1999; Cackett et al. 2007). The measurement of short lags between the X-ray emission and the optical, the leading of the X-rays whenever significant lags are determined, and the negligible optical inter-band lags, are all evidence that points towards reprocessing of the X-rays by the accretion disk, where the characteristic distances between the different emitting regions correspond to the lighttravel time. Also, light curves show that the amplitude of the X-ray emission is much larger than that seen in the optical, which can be explained by the damping of the signal during the disk reprocessing. However, a full picture of the interplay between the X-ray corona and the disk might not be complete just yet, as new evidence seems to suggest.

Long-term (i.e, a few years), well-sampled multiwavelength monitoring of AGN is only available for a few sources (e.g., Breedt et al. 2010; Chatterjee et al. 2009; Shemmer et al. 2001; Giveon et al. 1999; Türler et al. 1999; Korista et al. 1995). Here we present results from our monitoring of two sources, NGC 3783 and MR 2251-178, over 3 years at X-ray, optical, and near-IR wavelengths. NGC 3783 is a well-studied nearby $(z=0.0097)$ type 1 Seyfert galaxy. Its black hole mass from reverberation mapping is $3 \times 10^{7} M_{\odot}$ (Peterson et al. 2004). MR $2251-178$ is considered a nearby $(z \sim 0.0640)$ quasar with an X-ray luminosity of $\sim 4 \times 10^{44} \mathrm{ergs} \mathrm{s}^{-1}$ in the $2-10 \mathrm{keV}$ energy band. No accurate black hole 

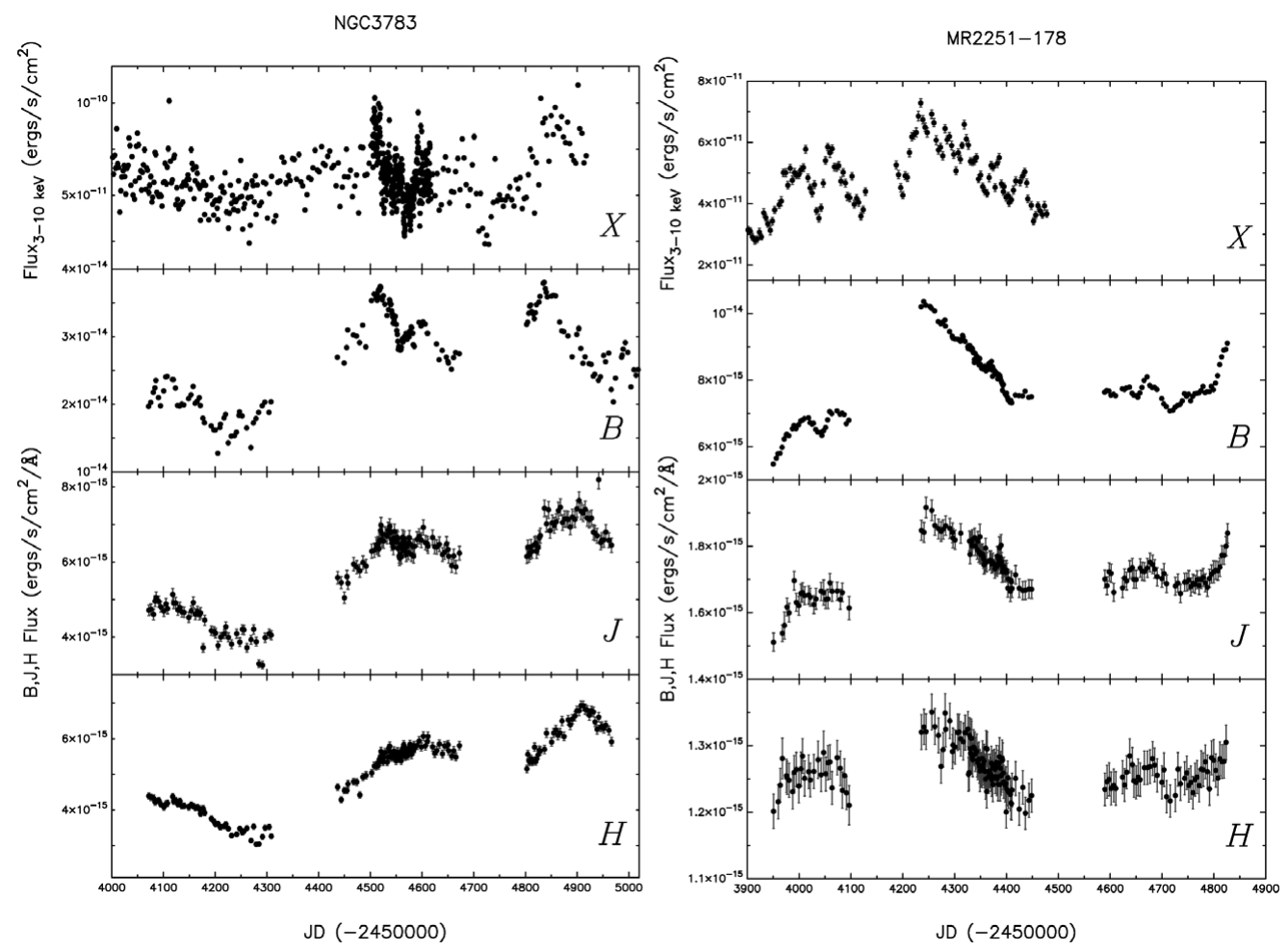

Figure 1. Three-year X-ray, optical, and near-IR data for NGC 3783 and MR 2251-178. An intensive period of monitoring for NGC 3783 occurs around JD 2454540 and for MR 2251-178 around $\sim 2454350$.

mass measurement exists for this object, but an estimate based on the characteristic break of its power spectrum suggests $\sim 10^{9} M_{\odot}$.

\section{X-Ray, Optical, and Near-IR Light Curves}

X-ray, optical, and near-IR data for NGC 3783 and MR 2251-178 were obtained using RXTE and the 1.3-m telescope located at CTIO and operated by SMARTS. The observing campaigns reported here extended from 2006 August to 2009 December. Details on the data acquisition and reduction are described by Arévalo et al. (2008, 2009) and Lira et al. (2010). The data presented here have not been corrected for host contamination within the apertures used $\left(\sim 7^{\prime \prime}\right.$ in the optical and $\sim 5^{\prime \prime}$ in the near-IR) and hence, the variability amplitudes only represent a lower limit to the real variations. Dilution by starlight might be particularly significant in the near-IR bands but is probably quite small in the optical.

Table 1. Cross-Correlation Analysis Results for NGC 3783 and MR 2251-178

\begin{tabular}{lcccc}
\hline Galaxy & $\boldsymbol{X} / \boldsymbol{B}$ lag & $\boldsymbol{B} / \boldsymbol{J}$ lag & $\boldsymbol{B} / \boldsymbol{H}$ lag & $\boldsymbol{B} / \boldsymbol{K}$ lag \\
\hline NGC 3783 & $6.6_{-6.0}^{+7.2}$ & $44.2_{-5.8}^{+4.9}$ & $67.7_{-3.3}^{+3.3}$ & $109.2_{-5.8}^{+4.9}$ \\
MR 2251-178 & $3.6_{-9.3}^{+9.3}$ & $9.0_{-3.5}^{+4.0}$ & $-2.1_{-4.4}^{+4.2}$ & - \\
\hline
\end{tabular}

All lags are expressed in days.

A positive lag between bands $Y / Z$ means that $Y$ leads. 

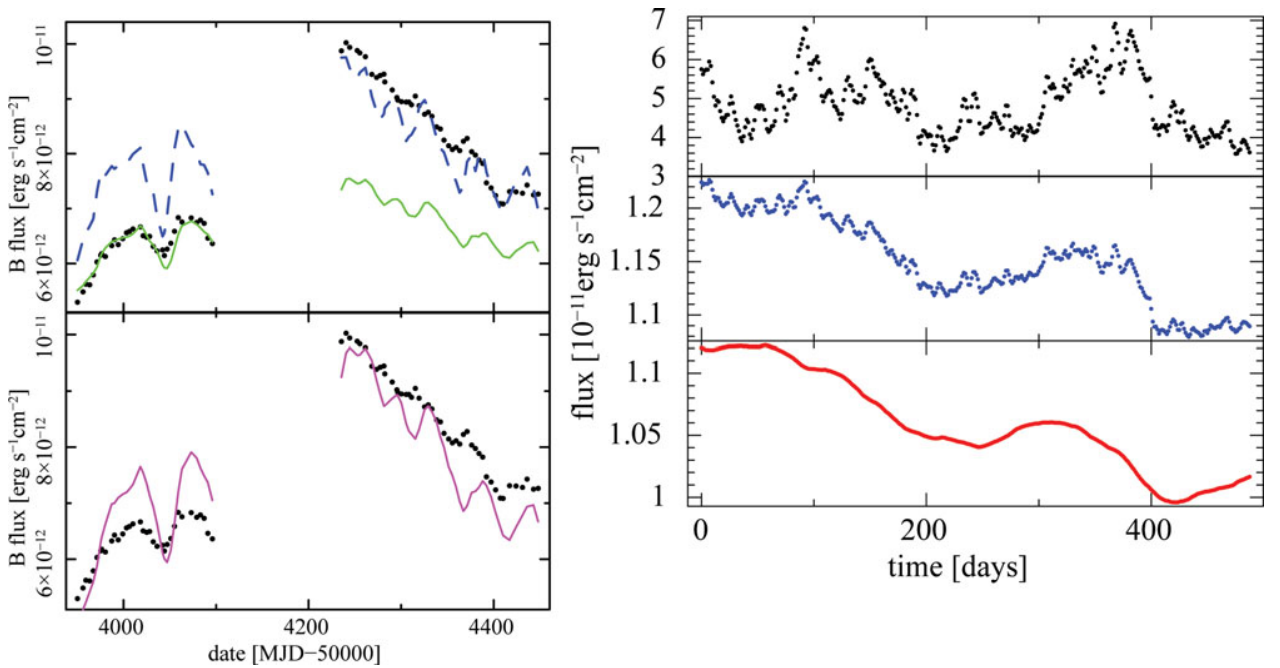

Figure 2. Left: Reprocessing model for MR 2251-178. The lines represent the reprocessed $B$-band light curves calculated using the observed X-ray light curves as input. The dots correspond to the observed $B$-band data. In the top panel, the reprocessing has been determined by fitting only one segment at a time: the solid line corresponds to the fit to the first segment of the light curve and the dashed line corresponds to the fit to the second segment. The solid line in the bottom panel corresponds to the best fit obtained when both segments are fited simultaneously. Right: Synthetic light curves for a propagating fluctuation model. The top panel represents the fluctuations in the innermost region, corresponding to the X-ray light curve. The bottom panel shows the $B$-band light curve for the same model. The middle panel shows the same $B$-band light curve when the effect of reprocessing the X-rays illuminating the disk has been taken into account.

Figure 1 presents the X-ray $(3-10 \mathrm{keV}), B, J$, and $H$-band light curves for NGC 3783 and MR 2251-178. A good correlation between the bands is readily seen. Cross-correlation analysis between the X-ray and $B$-band, and between the $B$ and near-IR bands was carried out. The results are summarized in Table 1. For details, see Arévalo et al. (2008, 2009) and Lira et al. (2010).

\section{X-Ray and Optical Variability Amplitudes: Evidence for Disk-Driven Variations}

Monitoring at X-ray, UV, and optical wavelengths has shown that variability is faster and larger amplitude at shorter wavelengths. In particular, the X-ray emission is always extremely variable. Our results for NGC 3783 and MR 2251-178, however, present a different behavior, only appreciated over long time scales: although the X-rays present the largest amplitude variations over time scales of days to weeks, the optical wavelengths show larger amplitudes of variation over time scales of months to years. These results are not compatible with the idea of X-ray reprocessing driving all the observed variability. Note that the lack of a correction for the host contamination would make this effect even more acute.

Another way to look at these results is presented in Figure 2, where we have plotted the two first segments of the $B$-band light curves for MR 2251-178. Here we have used a fully consistent reprocessing model where the X-rays illuminate the accretion disk (for details see Arévalo et al. 2008). The reprocessing follows a simple prescription where the incident X-ray flux is thermalized at each radius to the temperature $T(R)$ given by the 

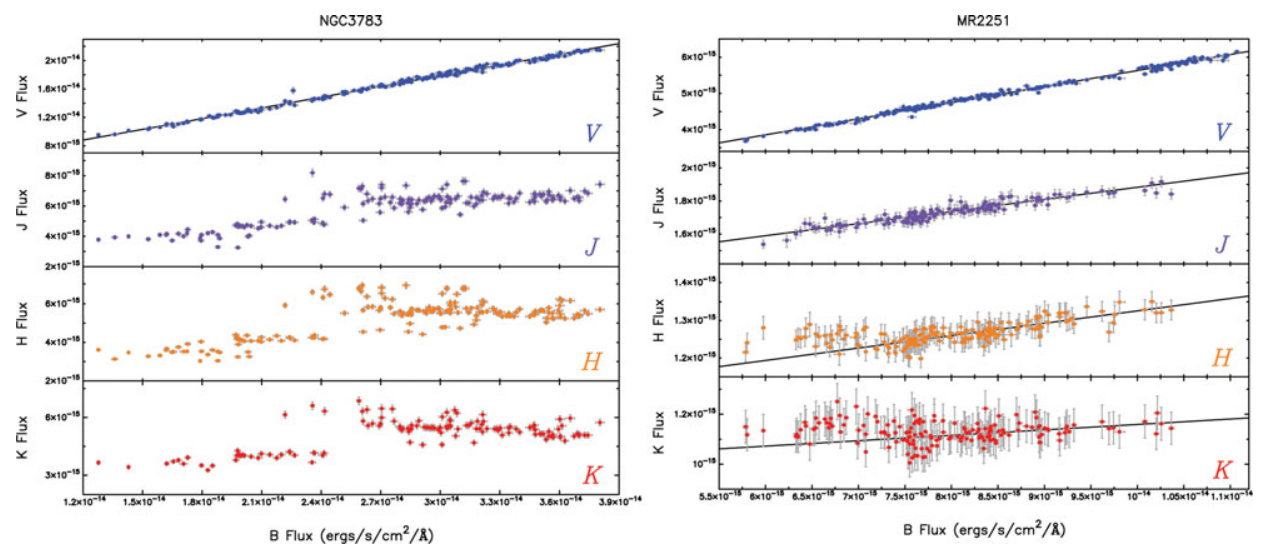

Figure 3. Optical-near-IR flux-flux plots for NGC 3783 and MR 2251-178.

thermal profile of an $\alpha$-disk. We used one of the two segments from the X-ray light curve and adjusted the parameters of the model in order to be able to reproduce the $B$-band light curve for the same segment. Then using the second segment of the X-ray light curve, the second $B$-band light curve was also determined without changing the model parameters. As can be seen in Figure 2, the amplitude variation between the first and the second segment of the X-rays light curves cannot reproduce the much larger jump seen between the two segments in the $B$-band. The bottom panel in Figure 2 shows the model of a reprocessed light curve if both $B$-band segments are fit simultaneously. Clearly reprocessing cannot explain both the large long-term trends and small short-term fluctuations.

It is possible to solve this inconsistency by introducing a second component to the variability: an intrinsic, slow, large amplitude variability from the accretion flow. Arévalo et al. (2008) have presented a model where instabilities are fed into the accretion flow and then migrate inwards consistently with the disk viscous time scale. These variations are characterized by a smooth light curve at optical wavelengths, as can be seen in the synthetic light curve shown in Figure 2. As these variations reach the inner parts of the accreting flow they give rise to the rapidly varying X-ray emission, which in turn illuminates the accretion disk. The resulting optical light curve shows the properties of both varying processes, the intrinsic variability of the accretion flow and the reprocessed variability of the X-ray corona. Results from the cross correlation between the model $\mathrm{X}$-ray and $B$-band light curves demonstrate that it is the short-term variability arising from reprocessing which dominates the measured lags, in good agreement with the observations.

An unexpected result comes from the $\sim 6$ day lag of the $B$-band behind the X-ray emission in NGC 3783. A similar result is also shown by the $V$-band light curve, which is not reported here (for details see Arévalo et al. 2009). For a black hole mass of $3 \times$ $10^{8} M_{\odot}$ in NGC 3783 (Peterson et al. 2004), a distance of 6 light days corresponds to $\sim 3500$ gravitational radii $\left(R_{g}\right)$, while the peak of the disk-related $B$-band emission for a canonical $\alpha$-disk corresponds to $\sim 1000 R_{g}$. The nature of this reprocessor and the amount of flux it contributes to the total emission in the $B$ band remain unclear. From our experience with cases where more than one source is responsible for the observed variability, it is extremely hard to disentangle from the available data the roles of the different contributors. 


\section{Near-IR Light Curves: Evidence for Cold Accretion Disks}

Suganuma et al. (2006) determined optical and near-IR light curves for four Seyfert galaxies and found that the IR-wavebands lag behind the optical emission in good correspondence with the estimates for the distance at which dust would sublimate given the bolometric luminosity of the central source, implying dust reverberation from a dusty torus. The sublimation radius can be estimated as $R_{\mathrm{d}} \sim 0.4 L_{45}^{1 / 2} \mathrm{pc}$, where $L_{45}$ is the central source luminosity in units of $10^{45} \mathrm{ergs}^{-1}$. Hence a near-IR lag of $\sim 85$ days can be expected for NGC 3783, which is somewhere between the lags measured for the $H$ and $K$ bands, suggesting the detection of torus emission in this object. However, the very short lags measured for MR 2251-178 clearly indicate that the bulk of the near-IR emission does not come from the torus, but for some structure much closer to the central source.

Figure 3 presents the $B$ versus $V$ and $B$ versus near-IR flux-flux plots for NGC 3783 and MR 2251-178, i.e., these correspond to simultaneous observations in the optical and near-IR bands. Immediately it can be seen that the optical bands trace each other tightly in both objects, indicating that the bulk of the flux comes from the reprocessing of the X-rays, giving nearly simultaneous variations in the $B$ and $V$ bands.

However, NGC 3783 and MR 2251-178 show very different behavior when it comes to the near-IR fluxes. On the one hand, NGC 3783 shows some evidence for a linear correlation between the $J$ and $B$ bands, but this seems to completely disappear in the $H$ and $K$ bands, where the level of the infrared flux is completely independent of $B$, except for the apparent presence of two regimes: a high and a low state, with a threshold between the two given by a $B$-band flux of $\sim 2.5 \times 10^{-14} \mathrm{ergs} \mathrm{s}^{-1} \mathrm{~cm}^{-2} \AA^{-1}$. On the other hand, MR 2251-178 shows a remarkable correlation between the $B$-band flux and all the near-IR bands. For $H$ and $K$, again it is apparent that for low $B$-band fluxes a new regime kicks in, for which the linear correlation breaks down. The linear fits shown in Figure 3 for the $H$ and $K$ bands correspond to those determined for a $B$-band flux greater than $7.5 \times 10^{-14} \mathrm{ergs} \mathrm{s}^{-1} \mathrm{~cm}^{-2} \AA^{-1}$ and are significant at a $99.9 \%$ confidence level. It has to be remembered that these plots show the level of correlation for nearly simultaneous variability, and therefore the lack of correlation for NGC 3783 does not contradict the clear correlation observed in Figure 1 and the lags reported. Notice also that the progressive flattening of the correlations with wavelengths might be due partly to the lack of a host contamination correction.

The tight correlation seen in the flux-flux plots of MR 2251-178 clearly indicates that the near-IR emission comes from a region very close to those where the $B$ and $V$-band fluxes are produced, that is, from the accretion disk. In fact, this was already suggested by the very small lags observed between these bands (Table 1 ). In particular, the $J$-band flux seems to be dominated by the disk component for all levels of the $B$-band flux.

To our knowledge, this is the first time that near-IR emission has been detected from an accretion disk. This suggests that in some sources cold regions of the disk are exposed to X-ray illumination. Uttley et al. (2003) already pointed out that the nature of the diskreprocessed emission will depend on the location of those regions of the disk exposed to the X-ray illumination. They invoke results from X-ray binaries to argue that the size of the X-ray emitting region, expressed in units of $R_{g}$, is constant for all sources. Hence, the range of temperatures that characterize the reprocessed emission will depend on the thermal structure of the disk, which in turn is determined by the black hole mass and the accretion rate as $T \propto M^{-1 / 4}$ and $T \propto \dot{M}^{1 / 4}$, for $\dot{M}$ expressed in Eddington units. Therefore, for small accretion rates and large black hole masses, we expect a cold accretion disk, where the near-IR emitting region will be located at small values of $R / R_{g}$ 
and will be more likely to be exposed to a significant fraction of the X-ray flux. The opposite situation, a hot accretion disk, is expected for sources characterized by small black hole masses or large accretion rates.

\section{Conclusions}

Evidence points toward a complex interplay between the different emitting regions around an AGN black hole. Long-term, multiwavelength monitoring is proving to be the only tool that will lead to a more comprehensive understanding of the physics of the accretion flow and its connection with the X-ray corona. Our observations suggest at least two components to the variability, a slow disk related modulation of the accretion flow and the much faster variability of the X-ray emission that illuminates the disk. At the same time, we have shown that near-IR disk emission can be significant and that its presence is probably related to the thermal profile of the disk as established by the mass and the accretion rate of the sources.

\section{Acknowledgements}

PL acknowledges financial support from Fondap Proyect 1501003 and from Fondecyt 1080603. EB acknowledges financial support from a SALT-Stobie scholarship and the University of Southampton.

\section{References}

Arévalo, P., Uttley, P., Lira, P., Breedt, E., McHardy, I. M., \& Churazov, E. 2009, MNRAS, 397, 2004

Arévalo, P., Uttley, P., Kaspi, S., Breedt, E., Lira, P., \& McHardy, I. M. 2008, MNRAS, 389, 1479

Breedt, E., et al. 2010, MNRAS, in press

Cackett, E. M., Horne, K., \& Winkler, H. 2007, MNRAS, 380, 669

Chatterjee, R., et al. 2009, ApJ, 704, 1689

Collier, S., Horne, K., Wanders, I., \& Peterson, B. M. 1999, MNRAS, 302, L24

Giveon, U., Maoz, D., Kaspi, S., Netzer, H., \& Smith, P. S. 1999 MNRAS, 306, 637

Korista, K., et al. 1995, ApJS, 97, 285

Krolik, J. H., Horne, K., Kallman, T. R., Malkan, M. A., Edelson, R. A., \& Kriss, G. A. 1991, ApJ, 371, 541

Lira, P., Arévalo, P., Uttley, P., McHardy, I. M., \& Breedt, E. 2010, in preparation

Peterson, B. M., et al. 2004, ApJ, 613, 682

Shemmer, O., et al. 2001, ApJ, 561, 162

Suganuma, M., et al. 2006, ApJ, 639, 46

Türler, M., et al. 1999, A\&AS, 134, 89

Uttley, P., Edelson, R., McHardy, I. M., Peterson, B. M., \& Markowitz, A. 2003, ApJ, 584, L53 